\title{
Designification of Neurotechnological Devices through 3D Printed Functional Materials
}

\author{
Nathan J. Castro* and Dietmar W. Hutmacher*
}

Neurotechnology as a research area can be defined as the convergence of neuroscience and tissue engineering/regenerative medicine. Through directed global initiatives concentrated on a better understanding of the human brain, an exponential rise in innovative functional biomaterials for the symbiotic integration of man and machine has risen. Unfortunately, neurotechnology as with other disruptive technologies faces the daunting path of traversing the broad chasm between the bench (development of technology) and the bedside (application of technology). Clinical translation of medical devices intended to assess, monitor, and treat sensorimotor injuries must address a selection of design and functional constraints which are cost limiting from a traditional manufacturing perspective. As a highly versatile technology, 3D printing in combination with advanced functional materials can be used to manufacture scaffolds for tissue engineering the neural-prosthesis interface as well as devices with predesigned capabilities of integrating spatial and temporal data sets usable in a diagnostic and therapeutic context. Therefore, recent neurotechnological advancements will be discussed in the realm of 3D printed functional materials (>2013) with a focus on their potential for clinical translation.

\section{Introduction}

Neurotechnology unlike traditional reparative/regenerative neural tissue engineering (NTE) is defined as the application of devices or tools for the monitoring, assessment, and control of the nervous system. ${ }^{[1]}$ Although discrete in their goal, they should not be viewed as mutually exclusive as neurotechnology can benefit significantly by adopting NTE strategies and concepts. A synergistic interplay working toward a dynamically responsive symbiotic relationship between nature and technology focused on sensorimotor repair, integration, and control is a feasible goal.

As a research area, neurotechnology has been actively investigated for over 50 years beginning with the establishment of the Society of Neuroscience in 1969 and continued by directed governmental policies. Political directives beginning with the Decade of the Brain (1990s) raised public awareness of the

Dr. N. J. Castro, Prof. D. W. Hutmacher

Institute of Health and Biomedical Innovation

Queensland University of Technology

Queensland 4059, Australia

E-mail:nathan.castro@qut.edu.au; dietmar.hutmacher@qut.edu.au

The ORCID identification number(s) for the author(s) of this article can be found under https://doi.org/10.1002/adfm.201703905.

DOI: $10.1002 / \mathrm{adfm} .201703905$ benefits of a greater understanding of the human brain ${ }^{[2]}$ which was followed by a proposal emphasizing the need of global transdisciplinary studies. These efforts pushed the idea toward understanding the biological underpinnings of cognitive behavior ("The Decade of the Mind") ${ }^{[3]}$ ultimately leading to the establishment of two pivotal initiatives.

The year 2013 was a seminal period in the evolution of neurotechnology where both the European Commission (Human Brain Project (HBP)) and the United States of America (Brain Research through Advancing Innovative Neurotechnologies (BRAIN initiative)) commenced large-scale publicly funded endeavors aimed to "accelerate our understanding of the human brain" ${ }^{[4]}$ as well as "[foster] the development and application of new technologies [enabling] researchers to produce dynamic pictures of the brain that show how individual brain cells and complex neural circuits interact at the speed of thought." ${ }^{[5]}$ Although early criticisms of ill-defined direction and broadness ${ }^{[6]}$ stunted the progress of the HBP, collectively these two initiatives have the potential to not only expedite discovery but also catalyze innovation and motivate rapid translation of technologies toward the formation of new industries through total financial commitments of \$1.9B and \$4.5B for the HBP and BRAIN initiative, respectively. Even though we are nearing the midpoint of both ventures, it is premature to speculate the long-term return on investment, yet we are seeing increased interest and attention being directed toward this broad area in the 21st century.

For that purpose, White et al. ${ }^{[7]}$ recently published a review on clinical translation of neurotechnology within the context of developing holistic therapy concepts for psychological and behavioral disorders. The review centered on seven underlying principles a medical device should exhibit for clinical success: (1) "verifiable" or having a testable intended function; (2) "useful" to fulfil an unmet need; (3) "consistent" agreement between the clinical need and product design; (4) "reproducible" effects for a homogeneous user population; (5) "mechanism-driven" or targeting a specific pathophysiological process; (6) "complete" and tunable parameters to produce an expected result with minimal expense of resources; and (7) "deployable" or highly likely for wide-scale adoption. Undoubtedly these principles establish a systematic framework for clinical translation of neurotechnology with emphasis on effective, targeted 
Brain-Machine Interface

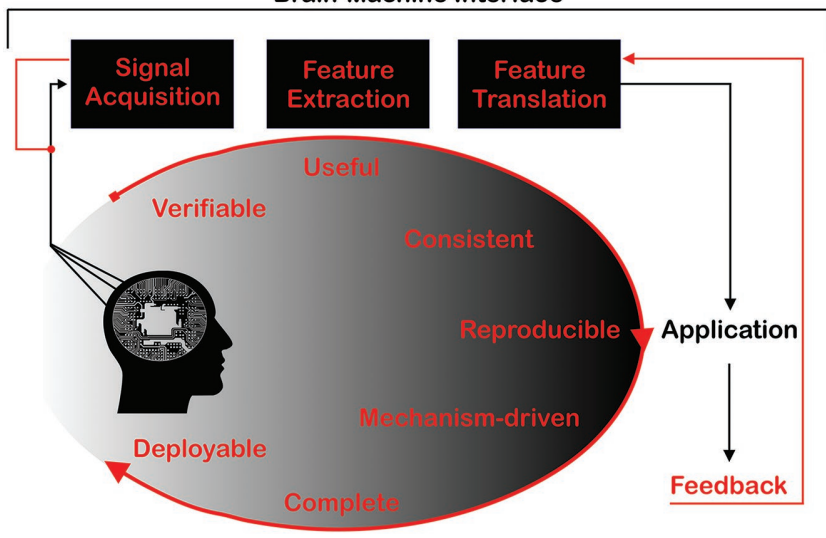

Figure 1. Design and systematic framework of a brain-machine Interface system. Signals produced by the brain are recorded from the scalp, from the cortical surface, or from within the brain. These signals are then analyzed and correlated to the patient's intent. These features are then translated into commands that control application devices that replace, restore, enhance, supplement, or improve natural CNS outputs.

and efficient neurotechnological device design as shown in Figure 1. An "idealized" neuroprosthesis would involve developing a closed-loop brain-machine interface (BMI)-controlled wireless prosthesis with self-learning for peripheral nerve signaling through integrated biocompatible packaging in combination with noninvasive wearable electronics. In the following we will begin exploring such a scenario beginning with neural probes for signal acquisition.

Based on the aforementioned design principles, we will briefly review and critically discuss three main research areas: (1) BMI with an emphasis on functional neural probes; (2) implantable and topical flexible electronics; and (3) custom prostheses with haptic sensing. The overarching goal is to provide the reader with an understanding of the current state-ofthe-art of these areas with respect to 3D printable functional materials as well as a prospective on the feasibility of integrating all areas toward a modular BMI-directed neuroprosthesis. We first begin the discussion with an overview of neural tissue engineering and scaffold design from a developmental biology perspective, then concentrate on the BMI interface by highlighting functional materials in combination with additive biomanufacturing technologies (i.e., 3D printing (3DP)) for effective translation of technologies addressing the brainmachine interface.

\section{Neural Tissue Engineering}

The need for new approaches and materials was explicitly articulated in the US National Institutes of Health (NIH) BRAIN initiative where "innovative new concepts, delivery methods, or biocompatible materials are required to dramatically reduce the invasiveness of acute or chronic electrodes", combined with the need to develop "new in vivo electrical capabilities, such as chronic multiunit intracellular recording or high density electrodes." Interfacing devices with the nervous system requires the integration of directed control of nerve cell activity and

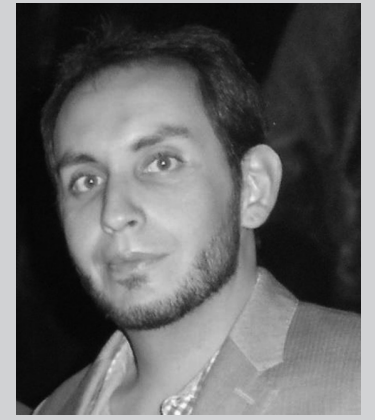

Nathan J. Castro is currently a Postdoctoral Research Fellow at Queensland University of Technology. He received his BS and MS degrees from the University of Texas at El Paso as part of the W.M. Keck Center for 3D Innovation and PhD from George Washington University within Lijie Grace Zhang's Bioengineering Laboratory for Nanomedicine and Tissue Engineering in 2016. He joined Prof. Hutmacher's group shortly thereafter with his current research interests involving multimaterial 3D printer development, tissue engineering and regenerative medicine, industry-driven international collaborations, and green chemistry photoinitiation.

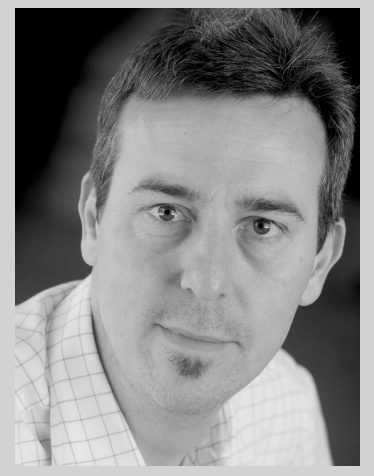

Dietmar W. Hutmacher is the Director of the Centre in Regenerative Medicine and Director of the Australian Research Council Centre in Additive Biomanufacturing at the Queensland University of Technology. He holds an MBA from the Royal Henley Management College and a PhD from the National University of Singapore. He is a pioneer in $3 \mathrm{D}$ printing

of Biomaterials, Tissue Engineering and Regenerative Medicine as well as "3D in vitro cancer models" and "humanized animal models." A number of endeavours have been successfully commercially translated under his mandate leading to five spin off companies.

acquisition of information with dynamic feedback regarding tissue activity. To achieve optimal performance from BMI/ brain-computer interface (BCI) devices, a long-term functional connection between "hard" device and "soft" excitable tissue is a condition sine qua non. First and second generation brain-machine/brain-computer Interface (BMI/BCI) devices use metal electrodes, which have a restricted ability to transfer electricity to biological tissues and a poor track record for longterm in vivo biocompatibility. This limits their employment in next-generation, high-resolution devices such as the bionic eye ${ }^{[8]}$ and brain-machine interfaces. Several biomaterials have been explored as coatings to improve the performance of metal electrodes, but scar tissue formation remains a major clinical problem.

For that purpose, functional materials and tissue engineered constructs (TECs) stand to play a vital role in the evolution of neurotechnology wherein the device is surgically connected and then remodeled in situ by the neural host tissue which 
subsequently facilitates the formation of neuromuscular junctions to record cell activity and drive peripheral prosthetics or rehabilitating limbs (via functional electrical stimulation). TECs provide a multitude of improvements when compared to current mainstream technologies including: being a better delivery system for neural cell stimulation; greater cytocompatibility for increased neural cell survival and tissue regeneration as well as facilitator of mid-to-late stage neural tissue hemostasis. TECs will also support the development of more complex BMI/BCI devices for the restoration of sensory and motor function.

Ideally, neural tissue should attach and expand upon the electrode surface to create an intimate junction between the device and host environment. Numerous strategies to mitigate scar tissue infiltration have been proposed, including drug delivery, ${ }^{\left[{ }^{[0]}\right.}$ flexible electrodes, ${ }^{[10]}$ and conductive polymer (CP) coatings. ${ }^{[11]}$ Recent advancements in NTE have led to a paradigm shift in biomedical electrode design. Technologies capable of 3D printing cell-laden biomimetic scaffolds (cellular solids) and/or functional matrices (hydrogels) to effectively support an integrated $\mathrm{BMI} / \mathrm{BCI}$ device with the surrounding tissue illustrate the possibility of a complete and deployable system.

\section{3D Bioprinting for Neural Regeneration}

Recently, NTE has shown great promise in addressing once perceived intractable acute neural injuries and congenital defects via the development of biomimetic hydrogels or "bioinks." Bioinks have shown to improve the development of functional network connectivity and neural synapses when compared to 2D cultures. ${ }^{[15]}$ In addition to the development of bioinks, 3D bioprinting/biofabrication technologies have spurred advancements in the field of customized 3D neural networks composed of matrices possessing suitable mechanical properties as well as biological complexity closely matching native neural tissues. An exemplary illustration of the potential of 3D bioprinted functional neural TECs was shown by Hsieh et al., ${ }^{[16]}$ where neural stem cells (NSCs) embedded within a novel class of waterborne polyurethanes were implanted in a traumatic brain injury adult zebrafish model leading to a recovery of mobility up to $81 \%$ when compared to untreated control. These findings suggest NSCs embedded within a suitable 3D hydrogel matrix may have the potential to improve the function of impaired nervous system in various neurodegenerative diseases.

In addition to functional bioink development, the combination of biomaterials and printing modalities toward complex 3D printed matrices with multifunctionality has also been explored. Lee et al. ${ }^{[17]}$ combined electrospinning and stereolithography bioprinting to manufacture composite scaffolds with enhanced neural cell adhesion and elongation properties illustrating not only the need but also the effectiveness of multimaterial technologies. These are only a handful of exemplary uses of 3D bioprinting for the replication of natural 3D bioprinted structures which illustrate the usefulness of 3D bioprinting/biofabrication for neural network models and functional scaffolds.

The reader is directed to Mir et al. ${ }^{[18]}$ for a thorough review focusing on 3D bioprinting for healthcare and medical applications where the current state-of-the-art in the field is presented with emphasis on the persistent limiting factors of biocompatibility, printability, and dynamically tunable available materials. Additionally, Kyle et al. ${ }^{[19]}$ critically reviewed the concept of "printability" as a driver toward the development of bioinks with special consideration placed on physicochemical characteristics.

Although NTE is an integral part of the discussion and advancements have been made in the area of 3D printable neural grafts ${ }^{[20]}$ and brain-like structures, ${ }^{[21]}$ the reader is directed to the following articles for in-depth and extensive reading of the state of the art of NTE.[22] Therefore, the authors' objective is not a comprehensive review of NTE, but to call attention to an oft over-looked body of evidence with direct implications in translational NTE and neurotechnology taking a perspective from the convergence of developmental biology and scaffold-based tissue engineering.

\subsection{Scar Formation and Neural Tissue Regeneration}

From an advanced biomaterials perspective it is extremely important to consider the complex interplay between the scaffold and early stage cell processes (attachment, proliferation, and differentiation) as well as extracellular matrix (ECM) development at various stages of the regeneration process which can take months to mature for neural tissues and their interfaces. In 2013, Ioannis Yannas put forward an evidence-based perspective for scaffold design combined with a biomaterial concept which focuses on a developmental process for tissue regeneration: tissue CBSIR. ${ }^{[12]}$ Two landmark reviews ${ }^{[13]}$ did lay out the rationale for what could be defined today as "scaffoldnomics." A very recent article has provided further empirical evidence substantiating the scaffold design and advanced biomaterial concept with respect to peripheral nerve tissue engineering. ${ }^{[14]}$

According to the CBSIR theory, which has refuted longstanding beliefs of scaffold design philosophy with respect to scar formation, scaffolds should not be designed as an exact copy exhibiting the properties of the tissue for which it is regenerating, but provide an interconnected porous architecture in combination with temporal mechanical stability founded on the biological understanding of control of wound contraction which is purported to result in the prevention of scar tissue formation to allow for guided tissue regeneration instead of tissue repair. Next, the rate of scaffold degradation after tissue maturation within the cellular solid and/or matrices should closely match the rate of new tissue synthesis. ${ }^{[23]}$ The CBSIR theory provides key evidence from an advanced biomaterials as well as basic cellular and biomechanical perspective on how to effectively design scaffolds in order to achieve the best possible regenerative effect.

Unfortunately, this concept is often overlooked by the tissue engineering community. Scaffold design considerations are complex with multiple aspects (3D structure, mechanical and surface properties, degradation kinetics/products, composition of biological components, etc.) and their changes over time in vitro and/or in vivo need to be taken into consideration. Therefore, a scaffold for neural tissue interface engineering has to be considered not as an inert 3D structure, but rather as a conduit for dynamic (chemical, biological, and mechanical) 
extracellular signaling, facilitating a developmentally conducive niche at the site of damage through progenitor cell recruitment, growth, and differentiation.

Developmental biology clearly shows that normal tissue healing in adults is a complex succession of progressive remodeling of pre-existing tissue structures that exhibit distinctively different mechanical and biological features. However, many neural tissue engineers have utilized scaffolds and matrices with mechanical and biochemical functions designed to closely mimic the properties of the final tissue. Lessons learned from developmental biology suggest that we should concentrate on the designification of scaffolds that mimic/promote these metastable, remodeling tissues and enhance cell-cell and cell-matrix interactions instead of precisely engineering a scaffold using the final tissue as a one-to-one design template. ${ }^{[24]}$ Not solely limited to "traditional" NTE scaffold design, the preceding considerations must also be applied to all aspects of a neurotechnological device. ${ }^{[25]}$ In the following we will discuss the BMI/BCI and regions of the technology which need to be addressed.

\section{4. $\mathrm{BMI} / \mathrm{BCl}$}

One of the primary roles of the central nervous system (CNS) is to transduce environmental (external) and physiological (internal) events in to intelligible and actionable signals. One way a BMI/BCI facilitates this process is by encoding/ decoding CNS-derived signals for direct control of electromechanical systems. CNS signals and activity are typically measured and converted into an artificial output that replaces, restores, enhances, supplements, or improves a natural CNS output thereby altering the dynamic interactions between the CNS and its external or internal environment. ${ }^{[26]}$ To further understand the limitations of current technologies, one needs to critically review the state of the art of advanced materials and devices used to record said activities.
CNS signals can be of electrophysiological, ${ }^{[27]}$ neurochemical, ${ }^{[28]}$ and metabolic origin ${ }^{[29]}$ encoding and transducing sensory and stimulatory responses that occur continually in the CNS. Most of these can be detected and monitored by measuring electric or magnetic fields ${ }^{[30]}$ or other parameters. Signal detection occurs with topical sensors placed on the scalp (electroencephalography), upon the surface of the brain (electrocorticography), or within the brain (neural probes) (Figure 2). A BMI/BCI records these signals, extracts pertinent measurements, and converts (or translates) them into new artificial outputs that actively or passively act on the environment or on the body itself. The entire process starts with acquisition or detection of a measurable signal emanating from a targeted region of interest and then transduces this signal to a voluntary action. Accurate and precise acquisition is imperative for short and long-term functionality of any BMI/BCI. Inherent spatial and temporal signal resolution is associated to each method of signal acquisition as electromagnetic signals quickly weaken as they propagate toward the scalp. Appropriate selection of detection method/device for use in a particular circumstance is contingent on reaching a balance between the feasibility of signal acquisition, the longevity of the implant, and the resolution required for proper transduction. Signal strength is inherently strongest closer to the source. Invasive neural probes exhibit excellent spatial delivery and acquisition of signals (single-cell/neuron) and have been widely used in stimulatory ${ }^{[31]}$ as well as single cell recording/ stimulating devices. ${ }^{[32]}$ Therefore, in the following we will focus on intracortical neural probes.

\subsection{Neural Probes}

Invasive electrophysiologic acquisition of brain signals is primarily accomplished by neurosurgical implantation of electrodes within or upon a patient's brain. The preferred

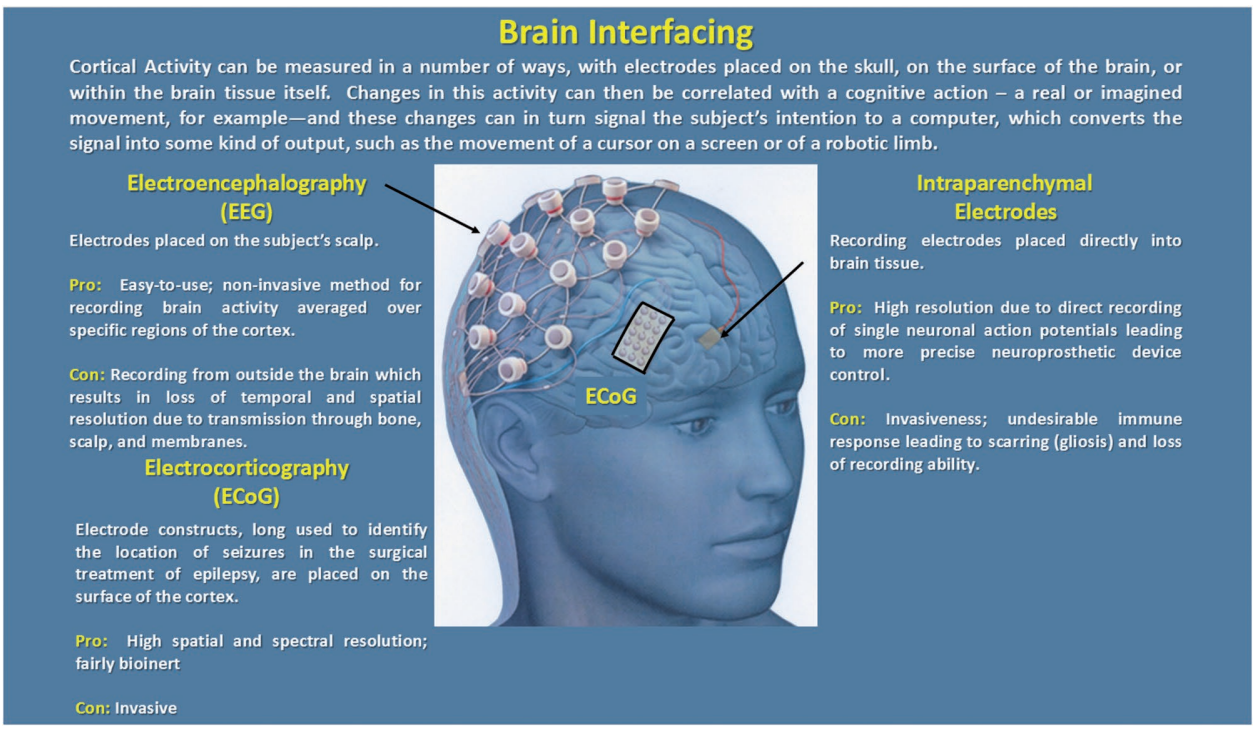

Figure 2. Brain-machine interfacing. The graphical illustration evaluates the benefits and drawbacks of invasive and noninvasive neural recording devices. Reproduced with permission. ${ }^{[33]}$ Copyright 2014, The Scientist. 
implantation site is the motor cortex since it is more easily accessible and generates signals that can be measured and generated through simple tasks such as actual or imaginary motor movements. ${ }^{[34]}$ Neural activity is not a single event, therefore continuous recording is necessary and typically undertaken using microelectrode arrays. These arrays are composed of single inorganic materials (also defined as neural probes) manufactured via traditional lithographic techniques. "Successful" recording is contingent on the spatial/temporal resolution of the desired signal, the number and placement of electrodes, and the functional lifetime of the device. ${ }^{[35]}$ Several electrode technologies have been developed and are shown in Figure 3. Some of which have effectively satisfied several of the aforementioned design principles previously discussed. Namely, those relating to verifiability, usefulness, and mechanism-driven where the intended use of some of these neural electrode microarrays is the transduction of signals designed to operate machine-driven systems (i.e., robotic arms) for paralytic patients.

As with all impactful translational work, international and interdisciplinary collaborations are imperative to the project's overall success. Collinger et al. ${ }^{[36]}$ provide an anecdotal example of such a project which lead to the development of a BMI-modulated neuroprosthetic arm. Although measurable clinical success has been realized with traditional microelectrode arrays, concerns regarding long-term implantation persist ${ }^{[37]}$ and research is being actively pursued in an effort to mitigate unwanted acute inflammation and chronic glial cell proliferation (gliosis) which contribute to signal loss/degradation and shortened device lifetime. ${ }^{[38]}$

In order for these devices to satisfactorily be complete and deployable, they should retain their intended function from years to decades with minimal active intervention resulting in a dynamically responsive self-correcting system. Signal loss has been attributed to acute immune response and long-term fibrous encapsulation of the electrode as a result of the presence of a foreign device. A recent in-depth study by Barrese et al. showed and elucidated multifactorial degradation of implants originally designed for permanent function ${ }^{[39]}$ via scanning electron microscopy analysis. Upon insertion of the electrode, vascular disruption ensues as well as destruction of all neurons in its path which initiates an acute immune response resulting in the recruitment of glial cells, ${ }^{[40]}$ which results in gliosis

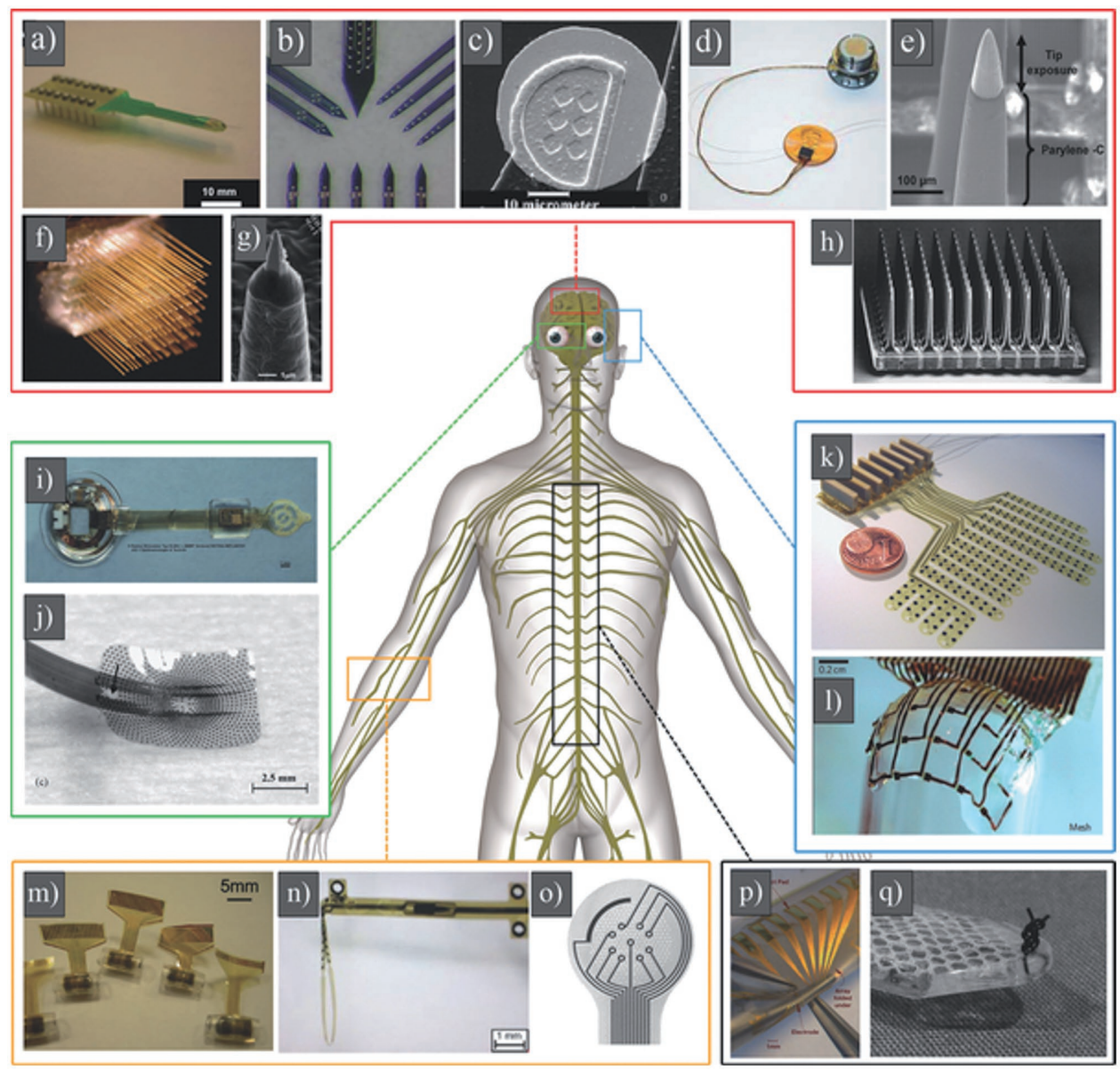

Figure 3. Neural probes and microarrays. Optical images of the eight-channel silicon substrate Michigan electrode A) and various site layouts B) with scanning electron image (SEM) imaging of individual gold site C). BrainGate microarray connected to Ti pedestal D), corresponding high magnification SEM E), and stacked arrays F). SEM of single gold-tipped parylene probe and array of $100 \mathrm{H}$ ). Epiretinal prosthesis encapsulated in parylene C/ silicon rubber I) with corresponding SEM J). Complete microelectrode array assembly K) and silk-supported polyimide electrode array molded upon a curved glass surface. Various microelectrode arrays for peripheral signal acquisition. Reproduced with permission. ${ }^{[35]}$ Copyright 2014 , WILEY-VCH. 


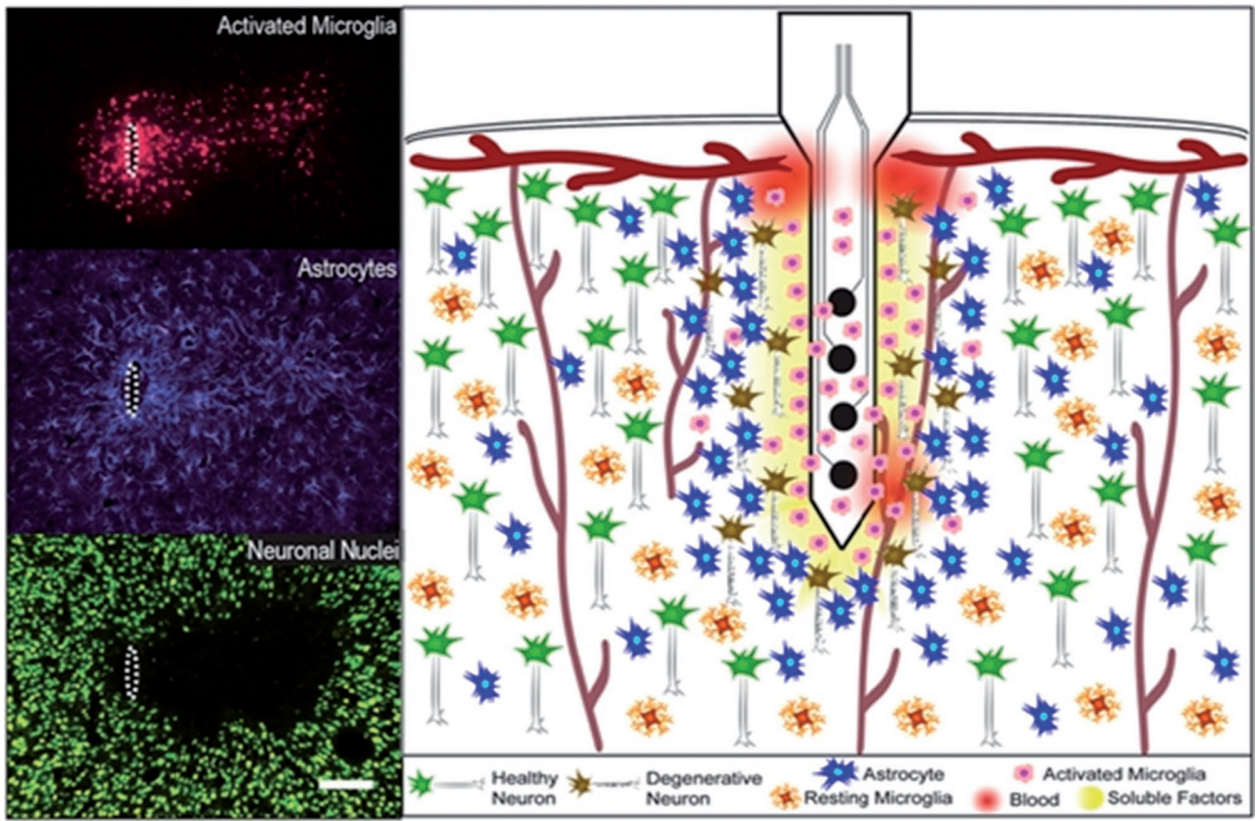

Figure 4. Acute neural tissue trauma due to neural probe insertion. Reproduced with permission. ${ }^{[41]}$ Copyright 2015 , IOP Publishing Ltd.

(fibrous encapsulation or scarring of neural tissue) occurring several months thereafter as shown in Figure 4.

Some of the contributing factors leading to long-term degradation include corrosion of metallic electrodes, loss of polymeric coating integrity and delamination of silicone substrates. In addition, mechanical property mismatch between the rigid electrode and soft tissue further compounds the problem from a clinical point of view. Although polymeric materials have been used in the production of soft microelectrode arrays, their potential in this long-term in vivo has yet to be fully realized. Therefore, we will discuss recent advancements in functional polymeric materials from a $\mathrm{TE} /$ regenerative medicine $(\mathrm{RM})$ perspective which may have the potential of mitigating issues related to compromised long-term in vivo biocompatibility and function.

One approach to address this is to modulate cell compatibility and tissue integration by incorporating NTE approaches toward electrode design. ${ }^{[42]}$ Seymour and Kipke evaluated the effects of subcellular lattice structures on tissue integration in a rat pial model using a polymeric substrate (Parylene-C). They could show the effectiveness of a fully interconnected porous architecture and its ability to minimize neuronal loss and decrease superficial microglia activation and protein deposition. In addition to electrode design, material selection should be considered in an effort to mitigate potential cytotoxic effects of metallic constituents commonly used for neural probes. For that purpose, conductive polymers have been investigated as functional materials for this particular application.

\subsection{Conductive Polymers and Organic Bioelectronics}

In the last two decades, there has been growing interest in the potential to develop incisive neural prostheses that interface selectively and favorably with the peripheral nervous system
(PNS) or central neural populations to supplement or replace body functions. These targeted input and/or output systems can be modulated to enhance neural communication between body systems or between the body and the environment. Organic electronics/bioelectronics ${ }^{[43]}$ is a research area bridging the gap between biology and technology. With the aforementioned TE/ RM design approaches, suitable materials with inherent biocompatibility for long-term tissue growth in combination with advanced manufacturing strategies, we are on the fringes of ever more useful technologies.

Conductive polymers (CPs) (Figure 5) are a class of organic polymers when in the presence of a dopant material form conjugated double-bond along their backbone. This reaction allows for ionic discharge which renders these polymers highly conductive and functionally analogous to semiconductors or metals. The unique caveat is the added capacity of tunability during the polymerization process. Notwithstanding, CPs also offer other tunable physicochemical properties, including hydrophilicity. Polypyrrole and poly(3,4-ethylenedioxythiophene) are two of the most widely used CPs synthesized for enhanced biocompatibility rendering them more favorable over inorganic conductive materials. Owing to the ease and low cost of processing the raw materials, these polymers have received greater attention. It is worth mentioning the two primary synthesis methods: chemical and electrochemical polymerization. In each case, radical cations are induced by oxidation and coupling to form dimers that propagate and polymerize by additional oxidization of the oligomer and/or coupling with the oxidized monomers. Specific to chemically mediated deposition, an oxidizing agent is required in addition to a large amount of polymer precursors as well as extensive reaction times.

Electrochemical processes induce polymerization by applying current through a system composed of CPs placed upon a conductive substrate. Typically, a working electrode or 


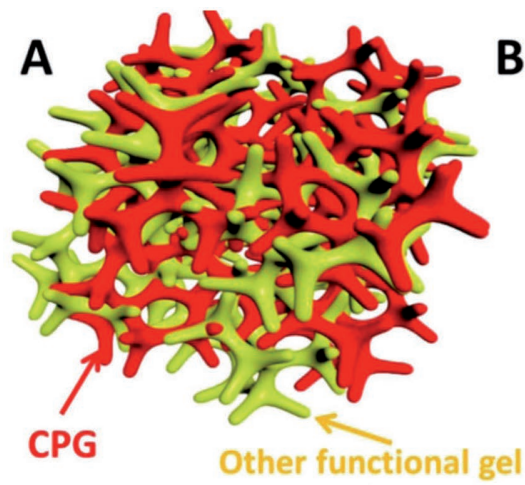

\section{B}
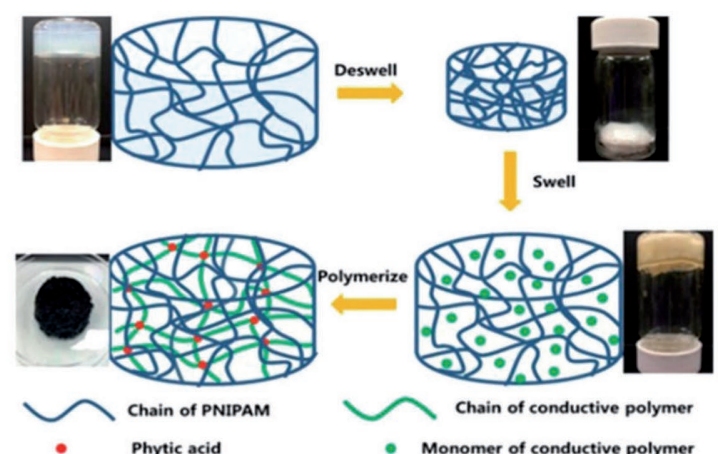

Figure 5. CPG/hydrogel interpenetrating network. A) Graphical representation of CPG/hydrogel interactions of an electrically conductive interpenetrating network. B) A thermoresponsive hydrogel consisting of a CPG crosslinked by phytic acid in poly (N-isopropylacrylamide) (PNIPAM). Reproduced with permission. ${ }^{[47]}$ Copyright 2017, American Chemical Society.

substrate is placed into a solution of precursor reagents (i.e., monomer and dopant) where an electric current is applied to facilitate oxidized cationic radicalization guiding deposition of $\mathrm{CP}$ on the substrate. ${ }^{[44]}$ Electrochemical polymerization is more effective when compared to chemical polymerization due to the need of less reagent material.

In order to harness the full capacity of TE/RM strategies to design functional neuroprostheses, CPs must transcend from thin-film patterning ${ }^{[45]}$ and conductive coatings to additive manufacturing technologies such as 3D printing. Since the first reported bioresorbable CP in 2002, ${ }^{[46]}$ few researchers have investigated rendering CPs 3D printable leading to functional electrically conductive bioactive TEC. Recently, Zhao et al. ${ }^{[47]}$ published an overview of work related to conductive polymer gels (CPGs) which combine the electrical conductivity of CPs with the tunability and manufacturability of hydrogels. The underlying behavior is analogous to the concept of interpenetrated networks $(\mathrm{IPNS})^{[48]}$ where a composite gel system exhibits characteristics of the individual components, but works synergistically for enhanced functional physicochemical behavior.

In the following we will highlight recent work related to electrically conductive stretchable electronics with an emphasis on manufacturing/3D printing for the development of TECdirected implantable electronics. Looking at both sides of the BMI-modulated neuroprosthesis, we will also expand the
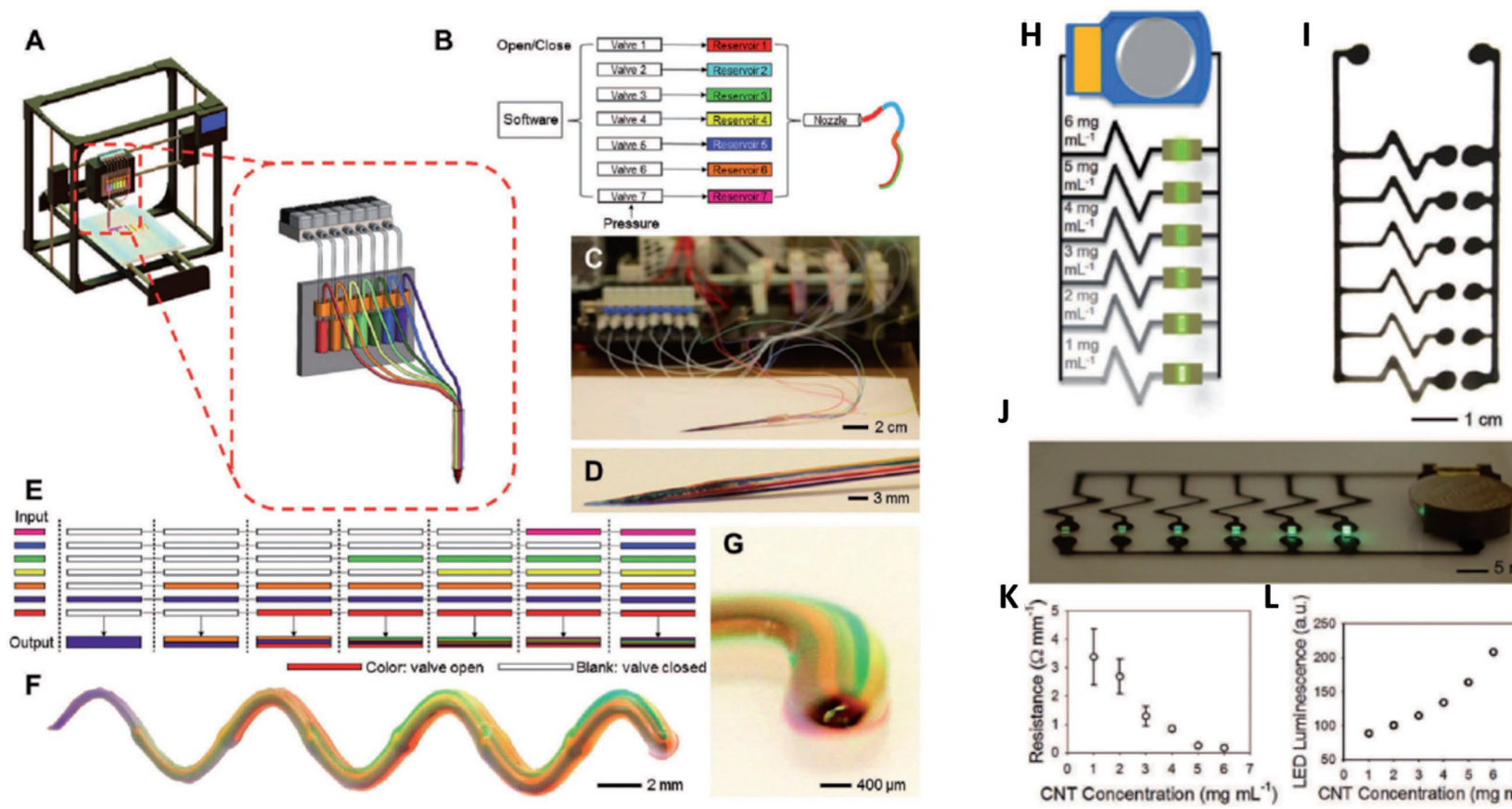

Figure 6. Continuous multimaterial extrusion bioprinter. A,B) Schematic illustration of mounted bioprinting head and multimaterial manifold with corresponding optical images (,D). Custom extrusion pattern E) and resultant printed multimaterial structure F,G). Graphical representation $\mathrm{H}$ ) and optical image I) of planar circuit with differential luminescence intensities L). M,N) Quantified resistance and luminescence of $2 \%$ alginate/carbon nanotube bioinks. Reproduced with permission. ${ }^{[51]}$ Copyright 2017, WILEY-VCH. 
discussion to include topical stretchable electronics for PNS signal transduction.

\section{3D Printed Implantable and Topical Stretchable Electronics}

Microelectrode arrays and neural probes are traditionally manufactured via lithographic techniques through serial deposition and etching of conductive metals and masking agents on planar substrates. Although conductive polymers have shown potential to integrate the desired properties of both discrete materials, extension to a fully extrudable and 3D printable matrix for rapid manufacturing is lagging. One of the primary limitations is the chemical and electrical process of incorporating dopant material(s). In most circumstances, incubation times on the order of several minutes are necessary to achieve the desired electrical properties. In addition to incubation time, material viscosity is also of concern where CPGs typically require heating for good flow. Wu et al. ${ }^{[49]}$ recently $3 \mathrm{D}$ printed a self-healing conductive hydrogel based on the chemically crosslinkable poly(2-acrylamido-2-methyl propane sulfonic acid) semi-IPN originally developed by Siddhanta and Gangopadhyay. ${ }^{[50]}$ Although defined and consistent extrusion was achieved after heating $\left(\approx 90{ }^{\circ} \mathrm{C}\right)$ and warm printing $\left(\approx 60{ }^{\circ} \mathrm{C}\right)$ with retained electrical conductivity, it was enhanced through the incorporation of activated charcoal. Unlike Wu's single material extrusion platform, Khademhosseini and co-workers ${ }^{[51]}$ developed a seven-material bioprinter with tunable mixing for graded and functional planar constructs (Figure 6).

To this day, few approaches and strategies have been developed to manufacture free-standing 3D printed electronics which satisfy all the above outlined NTE frontiers with respect to compliance, biocompatibility, and tissue remodeling. Due to the hydrophilic nature and low inherent viscosity of soft materials, self-supporting bioprinted structures are few and difficult to manufacture. Extensive studies investigating material properties including shear-thinning ${ }^{[52]}$ of advanced gellike materials and their effects on 3D bioprinting have been conducted, yet a few have been applied toward 3D printing of functional implantable electronics. One potential 3D printing strategy for free-standing/free-forming electronics has been illustrated by Bhattacharjee et al. ${ }^{[53]}$ (Figure 7) where granular gels consisting of Carbopol ETD 2020 polymer have been used as an embedding matrix for printing of high-resolution hollow interconnected structures with potential for use as biocompatible biosensors (Figure 7A-C). Although they have yet to realize a conductive 3D free-formed structure, studies have printed traditional substrate materials including polydimethylsiloxaone
A

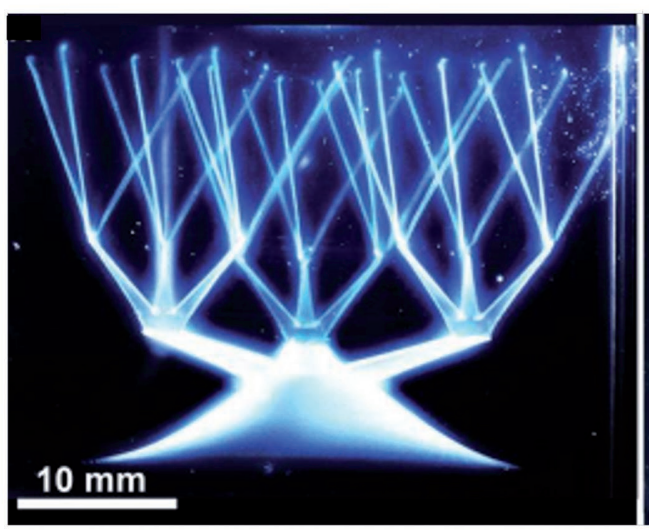

B

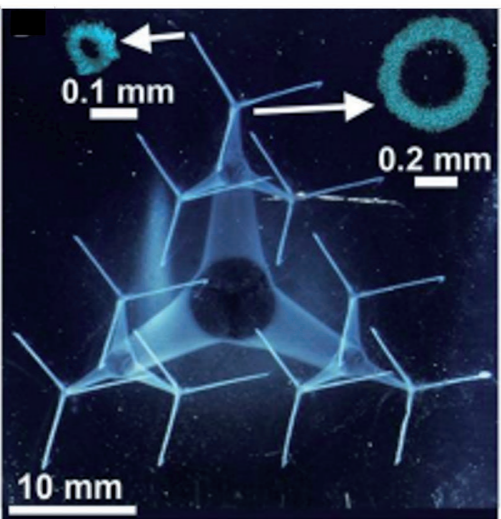

C

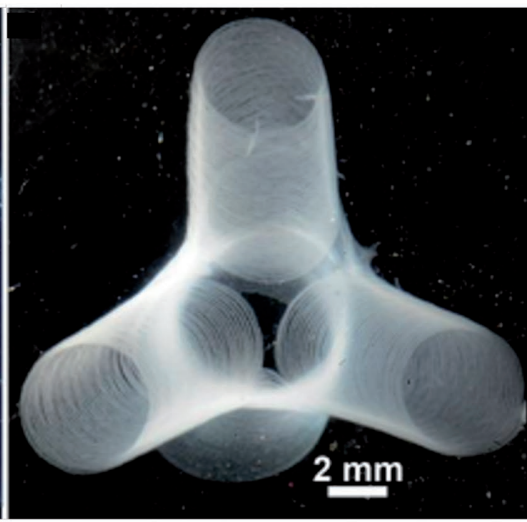

D

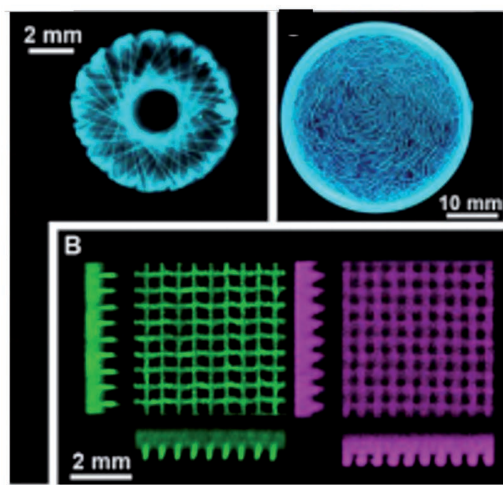

E

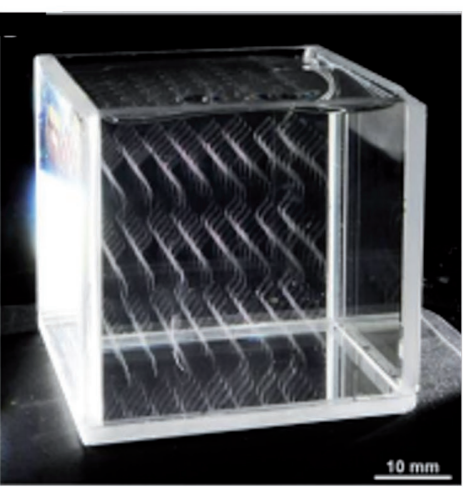

$\mathbf{F}$

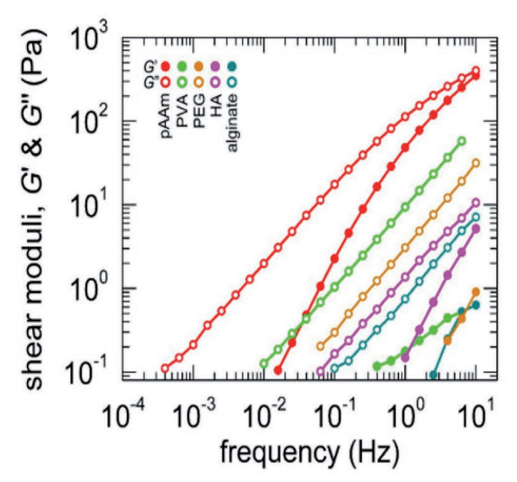

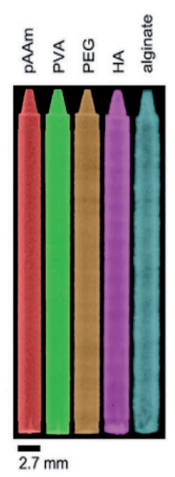

Figure 7. Free-form printing in granular gel. A-C) 3D printed interconnected hollow branched networks. D) Photocrosslinked linear arrays and corresponding skewed optical image E). F) Rheologically different biomaterials printed within a granular gel matrix. Reproduced with permission. ${ }^{\text {[3] }}$ Copyright 2017, American Association for the Advancement of Science. 
B

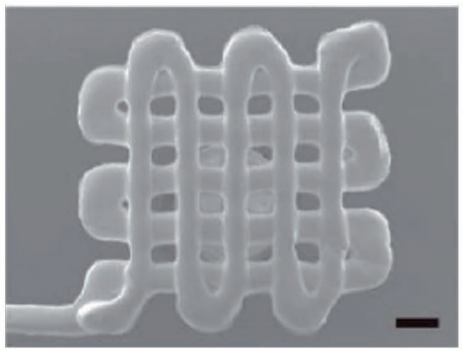

A

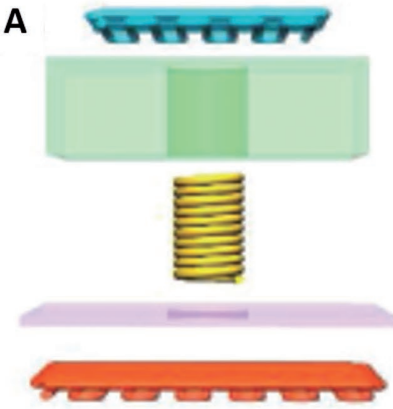

Top electrode

Supporting layer

Sensor layer

Isolating layer

Bottom electrode

Base layer

C
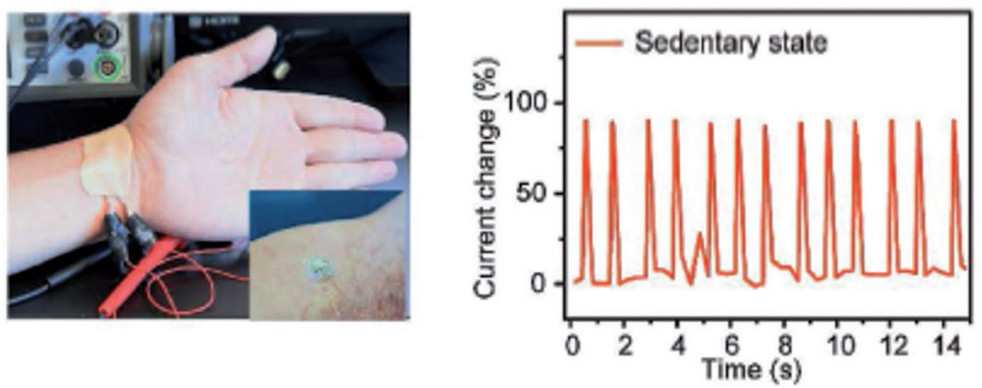

D

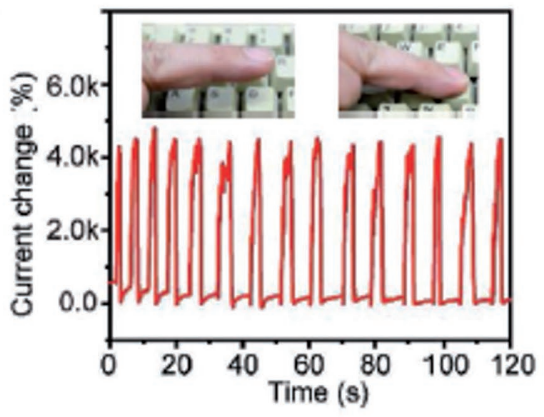

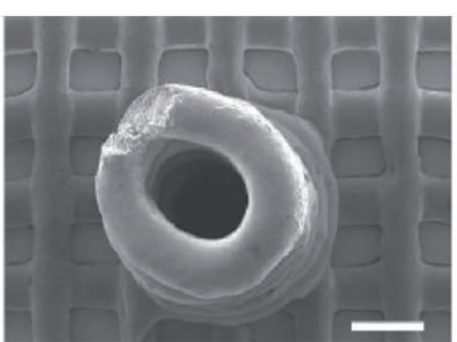
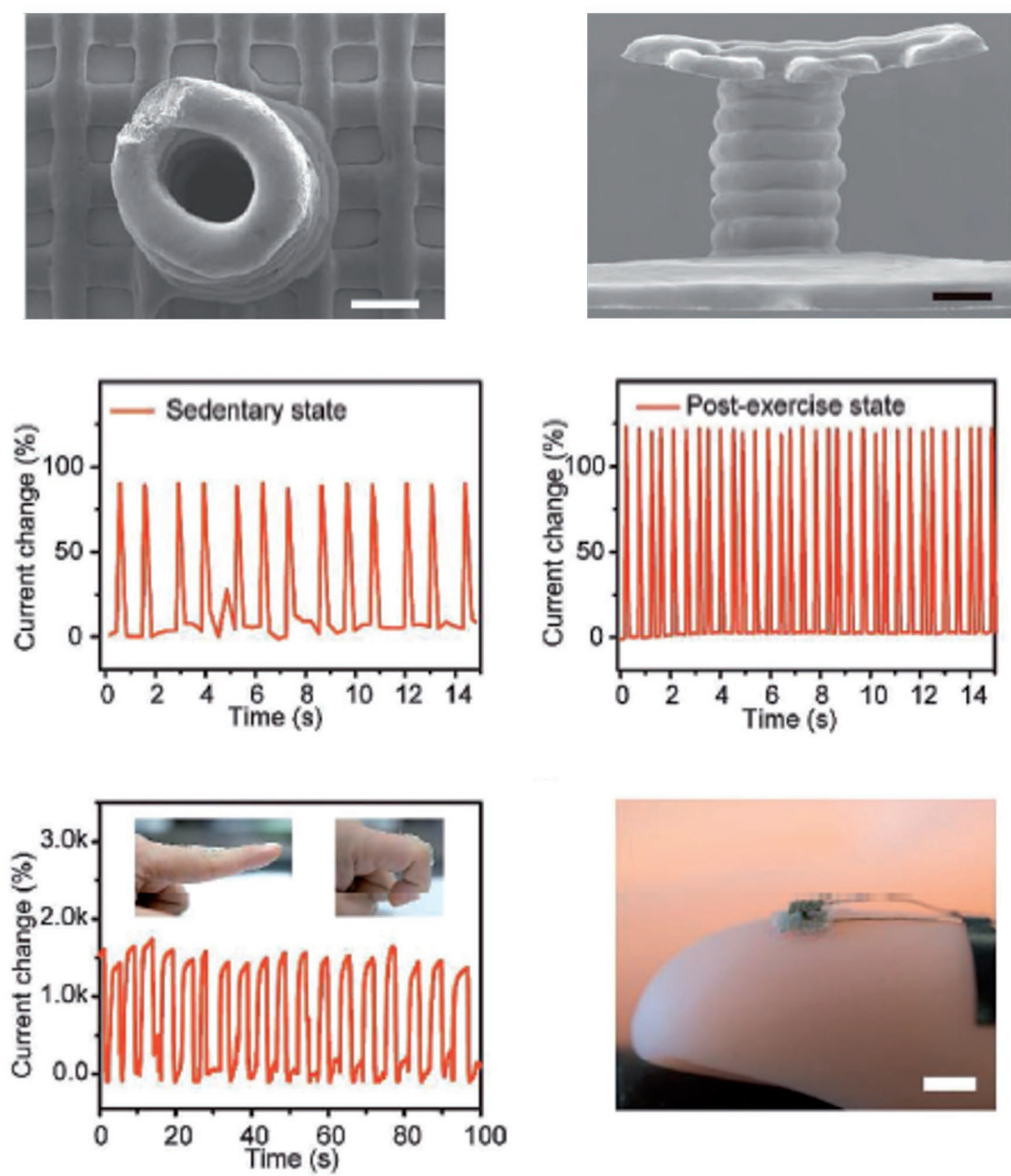

Figure 8. 3D printed tactile sensor. A) Schematic rendering of tactile sensor. B) Corresponding SEM images of top (left), coiled stretchable sensor layer (center), and full assembly (right). C) Mounted tactile sensor placed superficial to the radial artery (left) with corresponding resting pulse (center) and accelerated pulse postexercise (right). D) Representative current changes of mounted sensor upon pressing (left), bending (center) and mounted upon the fingertip. Reproduced with permission. ${ }^{[55]}$ Copyright 2017, WILEY-VCH.

(PDMS) as the granular gel. Notwithstanding, one can envision depositing dopant/monomer reagents within a granular gel containing undoped reagents for precise and controlled 3D electronics fabrication thus potentially satisfying a bulk of the requirements needed for implantable electrodes.
To further illustrate versatility and manufacturability, commercial photoinitiators and rheologically disparate biomaterials have also been incorporated to add shape and volume maintenance combined with biocompatibility of 3D printed structures (Figure 7D-F). 
Although improvements have been made in this area, further concerted efforts must be placed toward the development of evermore functional materials as well as bioprinting technologies which will allow for highly reproducible, long-term functional and deployable devices.

Considerable advancements have been made in the area of 3D printed wearable electronics. Although less rigorous design and material requirements are needed, wearable electronics must still exhibit certain characteristics including mechanical flexibility, scalability, integration with the dermis as well as be durable enough to endure day-to-day activities. Herein, we will focus on 3D printed wearable electronics with integrated haptic response for wireless BMI/BCI-control. Unlike transcortical neural probes which acquire afferent signals through intimate contact with individual neurons or neural networks, surface electromyography (SEMG) is traditionally used for signal acquisition by wearable electronics. In 2000 Hermens et al. ${ }^{[54]}$ published a seminal paper standardizing SEMG sensors which resulted in a list of parametric design considerations including electrode diameter $(<10 \mathrm{~mm})$, interelectrode distance $(<20 \mathrm{~mm})$ and sensor orientation (along long axis of muscle) with the goal of better signal-to-noise ratio and signal detection.

Recently, Guo et al. ${ }^{[55]}$ developed a 3D printed stretchable sensor for tactile signal acquisition (Figure 8). Briefly, the conductive ink was composed of submicrometer-sized silver nanoparticles embedded within a room temperature curable silicone elastomer ${ }^{[56]}$ and evaluated for extrudability at varying concentrations up to $86 \mathrm{wt} \%$. A sacrificial layer consisting of a curing retarder and thickening agent provided structural support for the overhanging top electrode.

In addition to $3 \mathrm{D}$ printing doped elastomeric materials, liquid metals have also been used in the development of tactile sensors as well as integrated antennae for wireless data transfer. ${ }^{[57]}$ As highlighted by Dickey, although liquid metals (i.e., gallium) are in abundance naturally they cannot be mined directly as they exist predominantly as impurities in other metals, therefore scalability is largely unattainable with these versatile materials.

\section{Clinical BMI/BCI-Modulated Neuroprosthesis}

As an exemplary illustration of clinical neurotechnology, Wodlinger et al. utilized two 96-electrode microarrays similar to those shown in Figure $3 \mathrm{~h}$ for signal acquisition and calibration of a 10 degree-of-freedom modular prosthetic limb (MPL) on a 52-year old tetraplegic patient up to $280 \mathrm{~d}$. The results illustrated accelerated learning of the patient with increasing MPL degrees of freedom ${ }^{[58]}$ as well as enhanced dexterity after virtual reality training. Therefore, emphasis has begun to be placed on the importance of visual context for proprioception ${ }^{[59]}$ as well as sensory feedback (i.e., haptic response) as an integral component of manual dexterity and object handling ${ }^{[58,60]}$ in BMI/BCI-modulated neuroprostheses. Through the use of 3D printing and functional materials, a wide range of possibilities exist for systematic integration of the distinct components related to neurotechnology. Although, we are far from realizing a technology which will satisfy all seven principles of clinical translation, much progress has been made. Standardization of testing protocols, 3D printing methods/technologies, and implementation are lacking and need to be addressed in order to move forward.

\section{Future Perspectives}

Genuine unmet medical needs persist in virtually every domain of brain and nervous system disabilities, including: Alzheimer's disease, addiction, anxiety, depression, epilepsy, multiple sclerosis, obesity, pain, Parkinson's, spinal cord injury, stroke, schizophrenia, sleep disorders, and traumatic brain injury. Henceforth, neuroscience is now moving from basic to translational science now defined as neurotechnology. In this feature article, we argued for the convergence of tissue engineering and its multidisciplinary disciplines into neurotechnology. When viewed separately, the technological innovations reviewed and discussed will not dramatically improve disease diagnosis and therapeutic effectiveness of brain and nervous system disorders. Therefore, investigating them unconnectedly would be an oversight. Initiatives such as BRAIN and the Human Brain Project spurred research in the fields of neuroscience and technology facilitating global collaborations. Further technological advancements purveyed through programs such as the Defense Advanced Research Projects Agency funded Neuro Function, Activity, Structure, and Technology (NeuroFAST) $)^{[61]}$ which highlight limitations with regard to real-time functional recordings with increased spatiotemporal resolution as well as the integration of functional and cognitive data within a closed-loop system. To drive the breakthrough treatments that will truly transform neurotechnology in the years to come biomaterial scientists, tissue engineers and neurotechnologists should be motivated to think through what will advance as the savoir faire to collaborate and build multidisciplinary teams which translate the innovations from bench to bedside.

\section{Conflict of Interest}

The authors declare no conflict of interest.

\section{Keywords}

3D printing, additive biomanufacturing, guided tissue regeneration, neurotechnology, tissue engineering

Received: July 13, 2017

Revised: August 29, 2017

Published online:

[1] J. J. Giordano, Neurotechnology: Premises, Potential, and Problems, CRC Press, Boca Raton 2012, p. 1-16.

[2] S. Ackerman, Institute of Medicine (U.S.), National Institute of Mental Health (U.S.), Discovering the Brain, National Academy Press, Washington, D.C. 1992.

[3] J. S. Albus, G. A. Bekey, J. H. Holland, N. G. Kanwisher, J. L. Krichmar, M. Mishkin, D. S. Modha, M. E. Raichle, G. M. Shepherd, G. Tononi, Science 2007, 317, 1321. 
[4] K. Amunts, et al. "The Human Brain Project: Creating a European Research Infrastructure to Decode the Human Brain." Neuron 2016, 92, p. 574-581.

[5] J. Sanes, C. Peña, BRAIN 2025: A Scientific Vision 2014.

[6] Y. Fregnac, G. Laurent, "Neuroscience: Where is the Brain in the Human Brain Project?" Nature 2014, 513, p. 27-29.

[7] S. W. White, J. A. Richey, D. Gracanin, M. A. Bell, S. LaConte, M. Coffman, A. Trubanova, I. Kim, Clin. Psychol. Sci. 2015, 3, 797.

[8] a) M. S. Humayun, E. de Juan Jr., G. Dagnelie, Ophthalmology 2016, 123, S89; b) D. V. Kaufman, ANZJ. Surg. 2016, 86, 635

[9] a) J. Y. Sim, M. P. Haney, S. I. Park, J. G. McCall, J. W. Jeong, Lab Chip 2017, 17, 1406; b) H. Shin, H. J. Lee, U. Chae, H. Kim, J. Kim N. Choi, J. Woo, Y. Cho, C. J. Lee, E. S. Yoon, I. J. Cho, Lab Chip 2015, 15, 3730; c) H. J. Lee, Y. Son, J. Kim, C. J. Lee, E. S. Yoon, I. J. Cho, Lab Chip 2015, 15, 1590.

[10] a) H. C. Lee, F. Ejserholm, J. Gaire, S. Currlin, J. Schouenborg, L. Wallman, M. Bengtsson, K. Park, K. J. Otto, J. Neural Eng. 2017, 14, 036026; b) X. Huijing, A. Weltman, H. Min-Chi, K. Scholten, E. Meng, T. W. Berger, S. Dong, IEEE EMBS 2016, 2016, 2806 c) S. A. Hara, B. J. Kim, J. T. Kuo, C. D. Lee, E. Meng, V. Pikov, J. Neural Eng. 2016, 13, 066020.

[11] a) H. S. Mandal, G. L. Knaack, H. Charkhkar, D. G. McHail, J. S. Kastee, T. C. Dumas, N. Peixoto, J. F. Rubinson, J. J. Pancrazio, Acta Biomater. 2014, 10, 2446.; b) V. Castagnola, E. Descamps A. Lecestre, L. Dahan, J. Remaud, L. G. Nowak, C. Bergaud Biosens. Bioelectron. 2015, 67, 450; c) H. Charkhkar, G. L. Knaack, D. G. McHail, H. S. Mandal, N. Peixoto, J. F. Rubinson, T. C. Dumas, J. J. Pancrazio, Acta Biomater. 2016, 32, 57 d) R. Green, M. R. Abidian, Adv. Mater. 2015, 27, 7620

[12] I. V. Yannas, Biomaterials 2013, 34, 321.

[13] a) D. W. Hutmacher, J. Biomater. Sci., Polym. Ed. 2001, 12, 107; b) D. W. Hutmacher, Biomaterials 2000, 21, 2529.

[14] I. V. Yannas, D. S. Tzeranis, P. T. C. So, Wound Repair Regen. 2017, 25, 177

[15] a) H. R. Irons, D. K. Cullen, N. P. Shapiro, N. A. Lambert, R. H. Lee, M. C. Laplaca, J. Neural Eng. 2008, 5, 333; b) W. Ma, W. Fitzgerald, Q. Y. Liu, T. J. O'Shaughnessy, D. Maric, H. J. Lin, D. L. Alkon, J. L. Barker, Exp. Neurol. 2004, 190, 276; c) N. Li, Q. Zhang, S. Gao, Q. Song, R. Huang, L. Wang, L. Liu, J. Dai, M. Tang, G. Cheng, Sci. Rep. 2013, 3, 1604.

[16] a) F. Y. Hsieh, S. H. Hsu, Organogenesis 2015, 11, 153; b) F. Y. Hsieh, H. H. Lin, S. H. Hsu, Biomaterials 2015, 71, 48

[17] S. J. Lee, M. Nowicki, B. Harris, L. G. Zhang, Tissue Eng., Part A 2017, 23, 491

[18] T. A. Mir, M. Nakamura, Tissue Eng., Part B 2017, 23, 245.

[19] S. Kyle, Z. M. Jessop, A. Al-Sabah, I. S. Whitaker, Adv. Healthcare Mater. 2017, 6, 1

[20] F.-Y. Hsieh, H.-H. Lin, S.-H. Hsu, Biomaterials 2015, 71, 48.

[21] R. Lozano, L. Stevens, B. C. Thompson, K. J. Gilmore, R. Gorkin, E. M. Stewart, M. in het Panhuis, M. Romero-Ortega, G. G. Wallace, Biomaterials 2015, 67, 264.

[22] a) F. L. Maclean, A. L. Rodriguez, C. L. Parish, R. J. Williams, D. R. Nisbet, Stem Cells Dev. 2016, 25, 214; b) X. Gu, F. Ding, D. F. Williams, Biomaterials 2014, 35, 6143; c) S.-J. Lee, W. Zhu, N. Castro, L. G. Zhang, in Neural Engineering: From Advanced Biomaterials to 3D Fabrication Techniques (Eds: L. G. Zhang, D. L. Kaplan), Springer International Publishing, Cham, Switzerland 2016, p. 1

[23] J. Henkel, D. W. Hutmacher, Bionanomaterials 2013, 14, 171.

[24] L. Stenberg, M. Stößel, G. Ronchi, S. Geuna, Y. Yin, S. Mommert, L. Mårtensson, J. Metzen, C. Grothe, L. B. Dahlin, K. Haastert-Talini, BMC Neurosci. 2017, 18, 53.

[25] C. H. Thompson, M. J. Zoratti, N. B. Langhals, E. K. Purcell, Tissue Eng., Part B 2016, 22, 125.
[26] F. Nijboer, et al. Design requirements and potential target users for brain-computer interfaces - recommendations from rehabilitation professionals, Brain-Computer Interfaces, Taylor \& Francis, Abingdon, Oxfordshire 2014, 1, p. 50-61.

[27] R. B. Campenot, Animal Electricity: How We Learned That the Body and Brain Are Electric Machines, Harvard University Press, Cambridge, MA 2016.

[28] N. Bunzeck, C. Thiel, Cortex 2016, 80, 161.

[29] E. Polo, S. Kruss, Anal. Bioanal. Chem. 2016, 408, 2727.

[30] a) B. B. Bartelle, A. Barandov, A. Jasanoff, J. Neurosci. 2016, 36, 4139; b) T. Kida, E. Tanaka, R. Kakigi, Front. Hum. Neurosci. 2015, 9, 713.

[31] G. Kook, S. W. Lee, H. C. Lee, I. J. Cho, H. J. Lee, Micromachines 2016, 7, 179.

[32] T. M. Fu, G. Hong, T. Zhou, T. G. Schuhmann, R. D. Viveros, C. M. Lieber, Nat. Methods 2016, 13, 875.

[33] E. C. Leuthardt, J. L. Roland, W. Z. Ray, in The Scientist, The Scientist, 2014.

[34] N. Naseer, K. S. Hong, Front. Hum. Neurosci. 2015, 9, 3.

[35] P. Fattahi, G. Yang, G. Kim, M. R. Abidian, Adv. Mater. 2014, 26, 1846.

[36] J. L. Collinger, M. A. Kryger, R. Barbara, T. Betler, K. Bowsher, E. H. Brown, S. T. Clanton, A. D. Degenhart, S. T. Foldes, R. A. Gaunt, F. E. Gyulai, E. A. Harchick, D. Harrington, J. B. Helder, T. Hemmes, M. S. Johannes, K. D. Katyal, G. S. Ling, A. J. McMorland, K. Palko, M. P. Para, J. Scheuermann, A. B. Schwartz, E. R. Skidmore, F. Solzbacher, A. V. Srikameswaran, D. P. Swanson, S. Swetz, E. C. Tyler-Kabara, M. Velliste, W. Wang, D. J. Weber, B. Wodlinger, M. L. Boninger, Clin. Transl. Sci. 2014, 7, 52.

[37] S. Waldert, Front. Neurosci. 2016, 10, 295.

[38] B. Gunasekera, T. Saxena, R. Bellamkonda, L. Karumbaiah, ACS Chem. Neurosci. 2015, 6, 68.

[39] J. C. Barrese, J. Aceros, J. P. Donoghue, J. Neural Eng. 2016, 13, 026003.

[40] D. J. DiLorenzo, J. Jankovic, R. K. Simpson, H. Takei, S. Z. Powell, Neuromodulation 2014, 17, 405.

[41] M. Jorfi, J. L. Skousen, C. Weder, J. R. Capadona, J. Neural Eng. 2015, 12, 011001

[42] J. P. Seymour, D. R. Kipke, Biomaterials 2007, 28, 3594

[43] R. M. Owens, G. G. Malliaras, MRS Bull. 2011, 35, 449.

[44] a) M. A. Sebaa, S. Dhillon, H. Liu, J. Mater. Sci.: Mater. Med. 2013 24, 307; b) S. Lehtimaki, M. Suominen, P. Damlin, S. Tuukkanen, C. Kvarnstrom, D. Lupo, ACS Appl. Mater. Interfaces 2015, 7, 22137.

[45] M. J. Yin, M. Yao, S. Gao, A. P. Zhang, H. Y. Tam, P. K. Wai, Adv. Mater. 2016, 28, 1394.

[46] A. N. Zelikin, D. M. Lynn, J. Farhadi, I. Martin, V. Shastri, R. Langer Angew. Chem., Int. Ed. 2002, 41, 141.

[47] F. Zhao, Y. Shi, L. Pan, G. Yu, Acc. Chem. Res. 2017, 50, 1734.

[48] J.-Y. Sun, X. Zhao, W. R. K. Illeperuma, O. Chaudhuri, K. H. Oh, D. J. Mooney, J. J. Vlassak, Z. Suo, Nature 2012, 489, 133.

[49] Q. Wu, J. Wei, B. Xu, X. Liu, H. Wang, W. Wang, Q. Wang, W. Liu, Sci. Rep. 2017, 7, 41566

[50] S. K. Siddhanta, R. Gangopadhyay, Polymer 2005, 46, 2993

[51] W. Liu, Y. S. Zhang, M. A. Heinrich, F. De Ferrari, H. L. Jang, S. M. Bakht, M. M. Alvarez, J. Yang, Y. C. Li, G. Trujillo-de Santiago, A. K. Miri, K. Zhu, P. Khoshakhlagh, G. Prakash, H. Cheng, X. Guan, Z. Zhong, J. Ju, G. H. Zhu, X. Jin, S. R. Shin, M. R. Dokmeci, A. Khademhosseini, Adv. Mater. 2017, 29.

[52] a) K. Markstedt, A. Mantas, I. Tournier, H. Martinez Avila, D. Hagg, P. Gatenholm, Biomacromolecules 2015, 16, 1489; b) C. B. Highley, C. B. Rodell, J. A. Burdick, Adv. Mater. 2015, 27, 5075; c) J. Malda, J. Visser, F. P. Melchels, T. Jungst, W. E. Hennink, W. J. Dhert, J. Groll, D. W. Hutmacher, Adv. Mater. 2013, 25, 5011. 
[53] T. Bhattacharjee, S. M. Zehnder, K. G. Rowe, S. Jain, R. M. Nixon, W. G. Sawyer, T. E. Angelini, Sci. Adv. 2015, 1, e1500655.

[54] H. J. Hermens, B. Freriks, C. Disselhorst-Klug, G. Rau, J. Electromyogr. Kinesiol. 2000, 10, 361.

[55] S. Z. Guo, K. Qiu, F. Meng, S. H. Park, M. C. McAlpine, Adv. Mater. 2017, https://doi.org/10.1002/adma.201701218.

[56] A. Frutiger, J. T. Muth, D. M. Vogt, Y. Menguc, A. Campo, A. D. Valentine, C. J. Walsh, J. A. Lewis, Adv. Mater. 2015, 27, 2440.

[57] M. D. Dickey, Adv. Mater. 2017, https://doi.org/10.1002/ adma.201606425.
[58] B. Wodlinger, J. E. Downey, E. C. Tyler-Kabara, A. B. Schwartz, M. L. Boninger, J. L. Collinger, J. Neural Eng. 2015, 12, 016011.

[59] J. E. Downey, J. M. Weiss, K. Muelling, A. Venkatraman, J.-S. Valois, M. Hebert, J. A. Bagnell, A. B. Schwartz, J. L. Collinger, J. Neuroeng. Rehabil. 2016, 13, 28.

[60] T. Tomlinson, L. E. Miller, Adv. Exp. Med. Biol. 2016, 957, 367.

[61] R. A. Miranda, W. D. Casebeer, A. M. Hein, J. W. Judy, E. P. Krotkov, T. L. Laabs, J. E. Manzo, K. G. Pankratz, G. A. Pratt, J. C. Sanchez, D. J. Weber, T. L. Wheeler, G. S. Ling, J. Neurosci. Methods 2015, 244, 52. 